\title{
A NEAR-INFRARED LED-BASED REHABILITATION SYSTEM: INITIAL CLINICAL EXPERIENCE
}

\author{
G. David Baxter ${ }^{1}$, Chris Bleakley ${ }^{1}$, Phil Glasgow ${ }^{1}$ and R Glen Calderhead ${ }^{2}$ \\ 1: Health \& Rehabilitation Sciences Research Institute, University of Ulster E Sports Institute for Northern Ireland, Northern Ireland, UK; \\ and 2: LG Biomedical, Tochigi, and Japan Phototherapy Laboratory, Tochigi, Japan.
}

\begin{abstract}
Treatment of sports injuries in top class athletes should be as fast and effective as possible, to enable the athlete to return to training or competition as quickly as possible so as to maintain as much of their preinjury muscle tone as they can. Laser therapy has offered an effective modality, particularly with deep-penetrating wavelengths in the near infrared around $830 \mathrm{~nm}$, but the treatment of a major muscle injury requires multiple point applications of a hand-held unit, and is very therapist intensive. A new hands-free operation system based on multipanels of near IR light-emitting diodes (LEDs, $830 \mathrm{~nm}, 180$ $\mathrm{mW} / \mathrm{cm}^{2}$ ) has been developed designed for applications in rehabilitation and physical therapy. The present study was designed to test the efficacy of this IR LED rehabilitation system (Omnilux Rehabilitation System, Photo Therapeutics, Ltd., Altrincham, UK) in a variety of sports injuries, both acute and chronic. Twenty-eight subjects were enrolled in the study with a variety of conditions: Joint Pain and Dysfunction $(n=8)$, Tendinopathies $(n=7)$, Muscle Pain and Dysfunction $(n=4)$, and Back Pain $(n=9)$. In 17 of the 28 subjects the results were excellent, and varied in the others. However, prudent doses of 54 and 108 $\mathrm{J} / \mathrm{cm}^{2}$ ( 5 and $10 \mathrm{~min}$ exposure times, respectively) were chosen. Higher doses may give better effects. Both acute and chronic pain types responded well to IR LED therapy. There were no adverse side effects, and all subjects were happy with the treatment. Practitioners were also pleased with the ease of use of the system, and found it safe and effective. Further controlled, double blind studies are required with larger populations and a greater range of pain entities to further examine efficacy with statistical analysis, but the results of the present efficacy study are very promising.
\end{abstract}

Key words: LED, IR phototherapy, tendinopathy, musculoskeletal pain, joint dysfunction

\section{Introduction}

Physical therapists are always looking for better systems to use for the treatment of injuries in their practice, including rehabilitation after injury. In sports medicine, the problem is compounded by the fact that the sportsman or sportswoman needs to get back into training as soon as possible after an injury so as to minimise the loss of muscle tone. Laser therapy (LLLT, LILT, et cetera) offered some powerful systems, particularly those in the infrared around the $830 \mathrm{~nm}$ wavelength, which could deliver a beam of comparatively high photon density deep into the target tissue, due to the comparative transparency of human tissue at that wavelength. Laser ther-

Addressee for Correspondence:

Professor David Baxter,

Health \& Rehabilitation Sciences Research Institute,

University of Ulster,

Jordanstown,

BT37 0QB,

Northern Ireland, UK

Tel: 02890368855 Fax: 02890368419

E-mail: gd.baxter@ulster.ac.uk apy systems are mostly used in a punctal fashion, however, which makes treatment of a large injury, such as in one of the major muscles, rather therapist intensive. Scanning lasers were developed to overcome this, but then the major advantage of high photon intensity was lost. Based on a spin-off from NASA space medicine, a new generation of very powerful light-emitting diodes (LEDs) was developed which had much higher and more stable output powers, better optics on the envelope, and perhaps most importantly, a quasimonochromatic output plus or minus only a few nanometres from the rated wavelength. Various wavelengths are now available, including $830 \mathrm{~nm}$. When LED's are precisely mounted in a panel array, taking into account the divergence of the individual beam so as to maximise the interference phenomenon, very high photon intensities can be achieved in the target tissue, allowing clinically useful photon intensities to reach comparatively deep into tissue. The present study was designed to assess the efficacy of a new therapeutic system with a multipanel array of $830 \mathrm{~nm}$

Manuscript received: March 2005

Accepted for publication: March 2005 


\section{ORIGINAL ARTICLES}

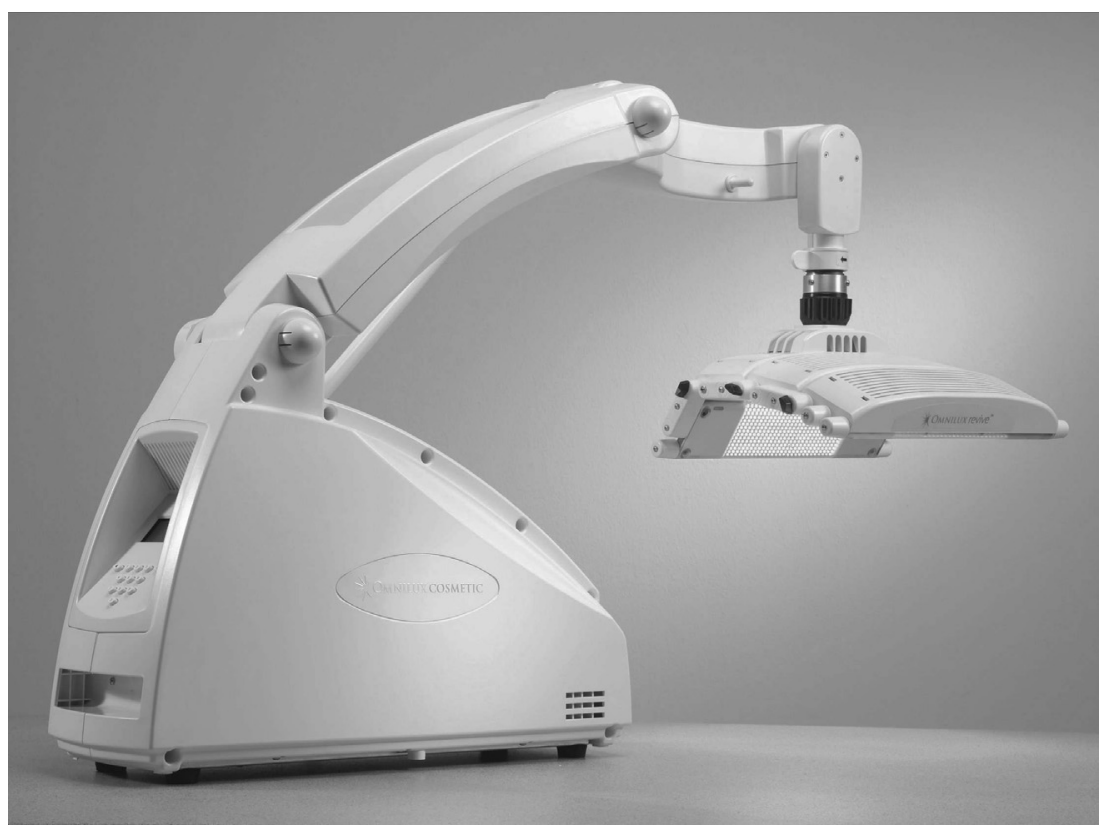

Fig 1: The Omnilux Rehabilitation System, consisting of an adjustable multipanel array of $830 \mathrm{~nm}$ LEDs on an articulated arm

LEDs in a variety of sports injuries in the physical therapy and rehabilitation environment.

\section{The System}

\section{Background}

The Omnilux Rehabilitation System is manufactured by Photo Therapeutics, Limited, Altrincham, UK, and is a monochromatic medium intensity near infrared therapy (MIMIRT) system, delivering $830 \mathrm{~nm} \pm 8 \mathrm{~nm}$, developed specifically for applications in physical medicine and rehabilitation. (Figure 1)

Benefits

The Omnilux Rehabilitation System aims to combine the benefits typically associated with laser therapy with mild heating effects associated with infrared therapy: its main features and advantages over alternative systems can be summarised as follows:

1: Significantly higher output than existing commercially available laser therapy systems $\left(180{\mathrm{~mW} \mathrm{~cm}^{2}}^{2}\right.$ over the treatment head; cf: a total of 500-700 mW for the best available multisource arrays)

2: Hands-free operation using a flexible, multi-positional array (Figure 2)

3: Safe mild heating with negligible risk of tissue burns (cf: infrared units)

4: Simple operation (especially when compared to laser therapy systems)

\section{Clinical Testing: Overview}

As the first stage in an integrated programme of research and development, the system was initially tested in an open trial under uncontrolled conditions in a sports rehabilitation clinic: the University of Ulster Clinic in Northern Ireland. This is based on the University's Jordanstown campus, and is the sports injury facility provided for the Sports Institute for Northern Ireland (see: www.uuclinic.ulster.ac.uk and www.sini.co.uk). This clinic is staffed by research-trained sports physiotherapists with extensive experience at national/international level in sports rehabilitation, and provides dedicated support to the elite athletes associated with the Sports Institute.

For this initial phase of testing, the unit was used in an open trial on a range of sports and musculoskeletal injuries, which would otherwise be considered as indicated for treatment with laser therapy using a multisource or 'cluster' array. All treatments for the current trial were performed using a conservative protocol of 5 or 10 minutes of treatment (equivalent to dosages of $54 \mathrm{Jcm}^{2}$ and $108 \mathrm{Jcm}^{2}$ ).

Over the course of five months, a total of just under 200 treatments with the Omnilux Rehabilitation system was performed on patients attending the clinic for a variety of sports injuries and musculoskeletal conditions, including tendinopathies, muscle tears and injuries, low back pain and joint pain. In all cases, treatment was performed as part of a comprehensive package of physiotherapy and rehabilitation.

\section{Outcomes}

\section{Users' Perceptions}

Feedback on the Omnilux Rehabilitation System was almost exclusively positive. Comments from therapists working with the Omnilux indicated the popularity of 

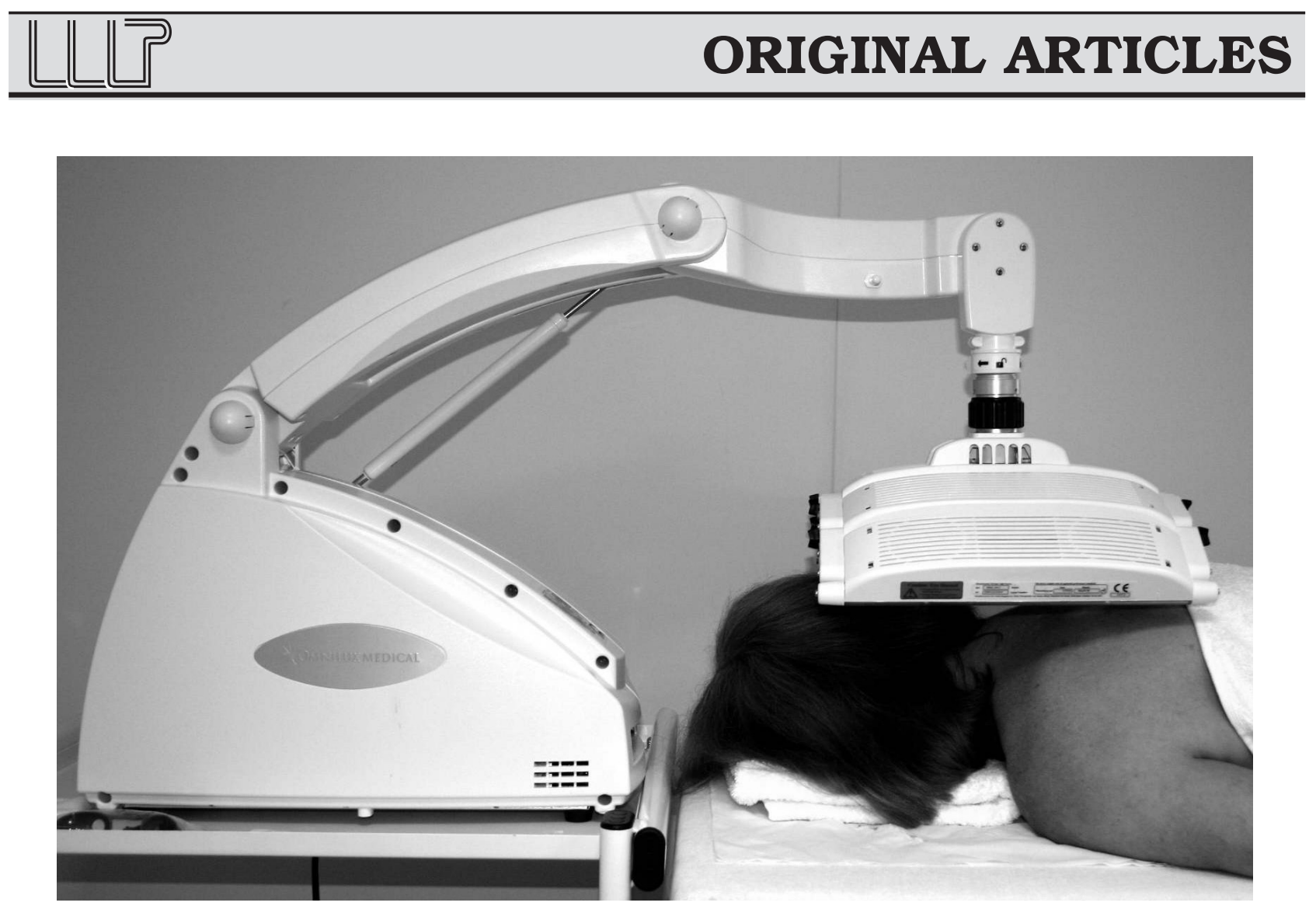

Fig 2: The Omnilux Rehabilitation System set up and in operation. The large multipanel array allows involvement of large areas in a hands-free manner. Here the $\mathrm{C} 1 / \mathrm{C} 2$ zone is being targeted, as well as the intervertebral entry and exit zones of $\mathrm{C} 3$ - about T6/7 in a dermatomal approach for intercostal pain.

the unit, largely due to its ease of use and 'hands free' operation, coupled with a early recognition of its potential to treat more deep-seated and intractable lesions and conditions. The only adverse comment on use of the machine related to the perception that the base unit might be potentially unstable to oblique forces (i.e. accidental knocks) in a busy clinical environment. However, this was considered to present more of a risk of damage to the machine, than of injury to the patient or therapist. For patients, treatment with the unit was typically found to be a pleasant experience, with the associated mild heating reported as relaxing.

\section{Safety}

The Omnilux Rehabilitation System was found to be safe in routine clinical application. The use of skin testing for hot and cold, usually an essential requirement for heating modalities, was not considered necessary. However, in larger studies this should be a prerequisite before the system can be used routinely in the clinical setting. Pre-clinical testing of the unit with irradiation times of up to and including 30 minutes (with the treatment head applied lightly 'in contact' to the exposed forearm) indicated that skin temperature reached a maximum (described as 'mild comfortable warmth') after 2-3 minutes, after which time temperature plateaued. No patient complaints were received during or immediately after application, nor were any adverse reactions reported.

\section{Initial Observations on Clinical Effectiveness}

In the context of the current work (i.e. an open, uncontrolled study) any attempt at definitive pronouncements on clinical effectiveness would be inappropriate. However, in summarising and analysing the experiences of the therapists who have used the Omnilux to date, it is possible to reach some preliminary recommendations on potential indications for treatment, and the most profitable direction(s) for future research and development. For this purpose, data were collected from clinical note review for those patients where follow up data were available, and where outcomes could be considered (as far as is possible) as reflecting the effectiveness of treatment with the Omnilux Rehabilitation System $(n=28)$. A variety of outcome measures were used, with the primary measure for each patient based upon what was most relevant (in the opinion of the treating therapist), as an indicator of improvement.

\section{Conditions Treated}

The range of conditions represented by this group are presented in Table 1, but can usefully be categorised as follows: Joint Pain and Dysfunction $(n=8)$, Tendinopathies $(n=7)$, Muscle Pain and Dysfunction $(n=4)$, and Back Pain $(n=9)$. When grouped in this way, it is 


\section{ORIGINAL ARTICLES}

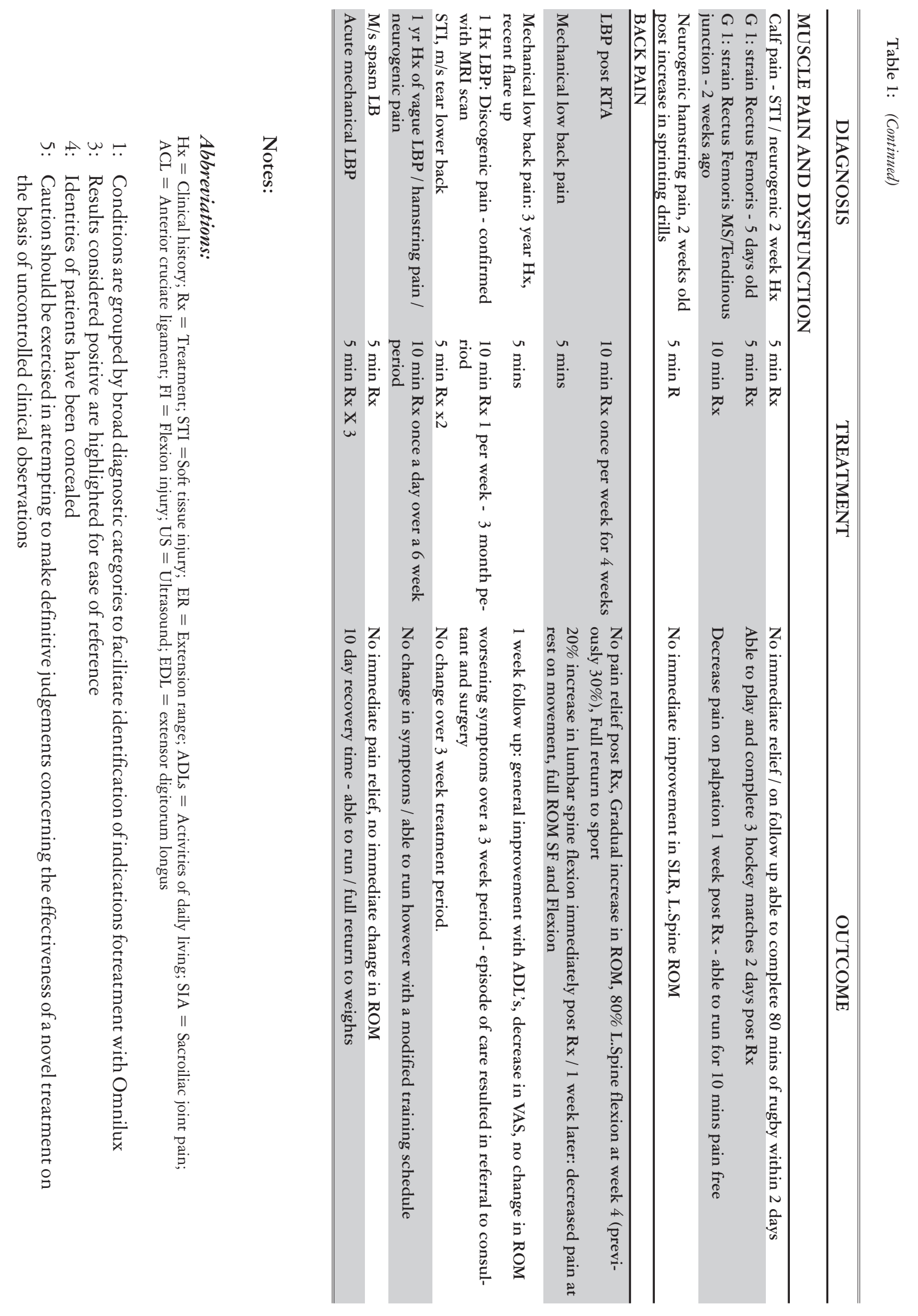




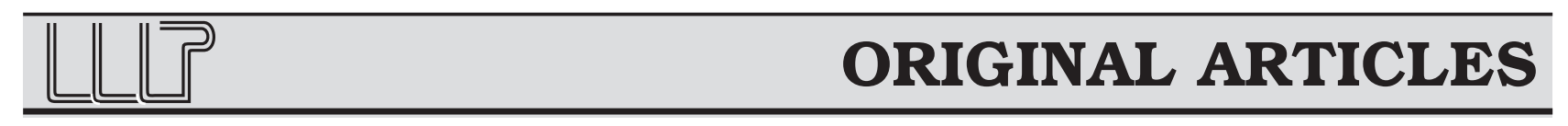

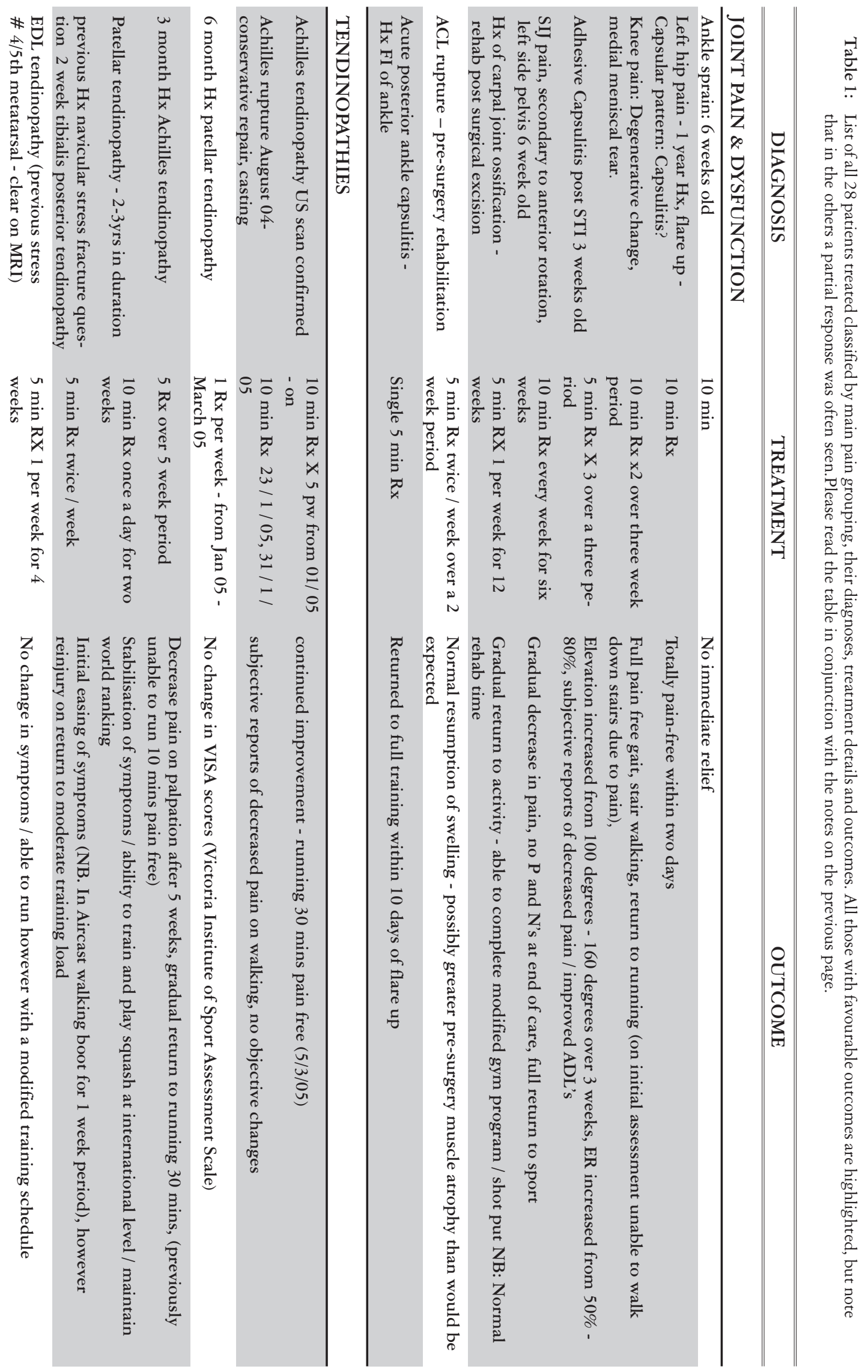


clear that the most consistently positive outcomes were achieved in two of these broad groups: Joint Pain and Dysfunction (6/8 demonstrating positive outcomes), and Tendinopathies (5/7). There were a range of conditions included in the former group, including cases of arthrogenic or joint pain $(n=3)$, capsulitis $(n=3)$, and ossification $(n=1)$. Perhaps significantly, the two cases where no clear positive outcome was achieved were associated with ligamentous injuries. For Tendinopathies, results indicated positive results with tendinitis affecting a variety of tendons of the lower limb (including the Achilles and patellar tendons); disappointing results were obtained in two cases, with no obvious pattern (patellar tendon and extensor digitorum longus [EDL] muscle).

More variable results were achieved in the treatment of Muscle Pain and Dysfunction (2/4 demonstrating positive outcomes) and Back Pain (4/9). While based upon small numbers $(n=4)$, results for myogenic pain and dysfunction would seem to indicate that simple muscle strain may potentially be suitable for treatment with the Omnilux Rehabilitation system; in contrast a neurogenic aetiology was diagnosed in each of the two cases of disappointing results. Similarly, for Back Pain, encouraging results for mechanical low back seemed to indicate potential benefits of treatment in such cases $(n=3)$; in contrast, treatment of back pain of other aetiologies (including discogenic pain, muscle tear etc) did not produce any consistent benefits.

\section{Dosage Parameters}

For this initial work, a cautious approach was used, based upon a simple (prudent) protocol of 5 or $10 \mathrm{~min}$ utes treatment ( 54 or $108 \mathrm{Jcm}^{2}$ ), with actual dosage at the discretion of the treating clinician. While the small numbers of cases to date precludes detailed analysis of the relationship between results and dosages employed, it would appear that the dosages used each produced positive benefits in patients. However, what is not clear is the degree to which higher dosages (based upon treatment times of up to 20 or even 30 minutes) may or may not produce superior or more consistently positive results.

\section{Discussion and Recommendations}

The current evaluation was undertaken as an initial clinical evaluation of the Omnilux Rehabilitation system in a specialised sports clinic serving the needs of the athletes supported by Sports Institute for Northern Ireland. The clinicians working with the system were highly experienced, and trained to postgraduate level: two had completed $\mathrm{PhDs}$ on physical agents (including laser therapy), and were also experienced in running clinical research trials. Furthermore, the patients treated were all athletes with a strong desire to return to competition or participation at the earliest opportunity. This makes for discerning research subjects who typically are not interested in pain relief per se, but rather the rather harder clinical end points of return to full training or participation: as a consequence perhaps the most relevant outcome measures for this trial were based upon functional measures (e.g. range of movement), or the time taken to return to a specified level of training (e.g. full running after two weeks).

While it was initially anticipated at the start of this study that best results would be achieved in more chronic or long standing conditions (anticipated because of the heating effects of treatment), participants noted that acute conditions, or acute exacerbations of recurrent conditions, also demonstrated positive outcomes. Thus chronicity does not seem to be an important determinant of treatment success with the system. Rather, therapists using the system soon found it to be useful in treating more deep-seated lesions which would otherwise not be amenable to treatment with other modalities (e.g. ultrasound or regular laser therapy).

It should be noted that the Omnilux Rehabilitation System was used alongside other treatment interventions, ranging from manual treatments to exercise prescription. Exclusion of other treatments would have raised ethical issues (with-holding of other effective treatments), not least in a facility that supports the rehabilitation of elite athletes. However the current approach did provide a more clinically relevant and realistic assessment of the effectiveness and potential role of the Omnilux Rehabilitation System in routine clinical practice. Future controlled trials with the system should ideally be based upon active and placebo treatment performed under blinded conditions, alongside a standardised package of 'best care' that is offered to all participants. A key element in this will be the design of some method of blinding for the machine operator.

Based upon the encouraging clinical results from this initial work, the following can be identified as the immediate priorities for research and development:

Increasing the clinical database on the Omnilux Rehabilitation system.

This would involve continuing with 'open recruitment' evaluations with the system at 2-3 centres with adequate patient throughput, to include an outpatient UK National Health Service (NHS) physiotherapy department (where there would be larger numbers, but more variable in terms of musculoskeletal conditions), as well as a sports clinic or private practice (where numbers would be smaller, but more limited in range of conditions). 


\section{Safety Testing.}

This preliminary work would indicate that the Omnilux Rehabilitation System is safe in routine clinical operation. However, and particularly given the potential use of dosages of up to 30 minutes, controlled testing of skin temperature profiles should be undertaken (proposed $\mathrm{n}=10-20)$, using a variety of skin types, at times of up to 40 minutes, to confirm the 'plateau' effect on skin temperature we observed in the current study. This would also provide useful data for technical and users' manuals, and should be incorporated into the first full publication on the device. This temperature testing could be completed alongside pilot work (within a 3 month period) at a single site; it would however require ethics permission and access to physiological recording systems.

\section{Pilot Studies for Controlled Trials.}

Based upon the data collected to date, two relatively common clinical conditions can be recommended as potentially useful indications for future clinical trials: Achilles tendinitis and lateral epicondylitis (tennis elbow). As a precursor to full blown trials in these areas, it is recommended that pilot/feasibility studies ( $n=5-10$ per treatment group) should be initiated as soon as is practicable. This work would be completed under controlled conditions, and could be single site and thus require one unit for its successful completion, over a six-month period. Again, ethics permission would be required for this work.

\section{Conclusion}

In conclusion, the Omnilux Rehabilitation System proved safe and efficacious in the treatment of a variety of sports injuries using a prudent 5 or 10 minute exposure time (respective radiant flux of $54 \mathrm{~J} / \mathrm{cm}^{2}$ and 108 $\mathrm{J} / \mathrm{cm}^{2}$ ). Subjects reported mild but pleasant heating in the treated area, and practitioners appreciated the ease of use of the system over large treatment areas in a hands-free operation. Further controlled, double-blind trials with larger patient numbers to enable the investigation of dose-dependent efficacy are warranted, in order to arrive at the optimum dosimetry and treatment technique. 